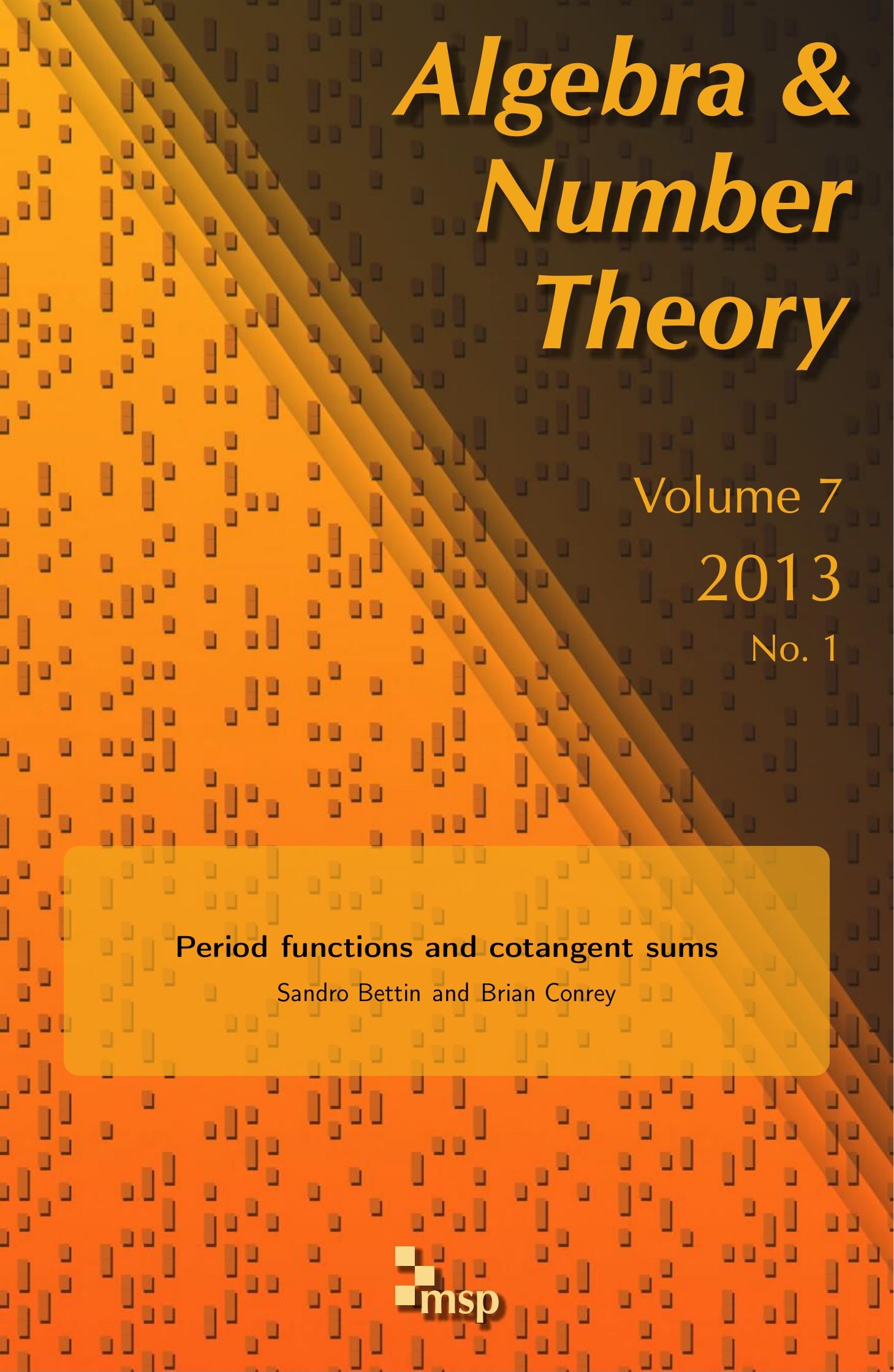




\title{
Period functions and cotangent sums
}

\author{
Sandro Bettin and Brian Conrey
}

\begin{abstract}
We investigate the period function of $\sum_{n=1}^{\infty} \sigma_{a}(n) \mathrm{e}(n z)$, showing it can be analytically continued to $|\arg z|<\pi$ and studying its Taylor series. We use these results to give a simple proof of the Voronoi formula and to prove an exact formula for the second moments of the Riemann zeta function. Moreover, we introduce a family of cotangent sums, functions defined over the rationals, that generalize the Dedekind sum and share with it the property of satisfying a reciprocity formula.
\end{abstract}

\section{Introduction}

In the well-known theory of period polynomials one constructs a vector space of polynomials associated with a vector space of modular forms. The Hecke operators act on each space and have the same eigenvalues. Thus, either vector space produces the usual degree $2 L$-series associated with holomorphic modular forms. Lewis and Zagier [2001] extended this theory and defined spaces of period functions associated to nonholomorphic modular forms, that is, to Maass forms and real analytic Eisenstein series. Period functions are real analytic functions $\psi(x)$ that satisfy three-term relations

$$
\psi(x)=\psi(x+1)+(x+1)^{-2 s} \psi\left(\frac{x}{1+x}\right),
$$

where $s=1 / 2+i t$. The period functions for Maass forms are characterized by (1) together with the growth conditions $\psi(x)=o(1 / x)$ as $x \rightarrow 0^{+}$and $\psi(x)=o(1)$ as $x \rightarrow \infty$; for these, $s=1 / 2+i r$, where $1 / 4+r^{2}$ is the eigenvalue of the Laplacian associated with a Maass form. For Eisenstein series, the $o$ 's in the growth conditions above are replaced by $O$ 's if $t \neq 0$ and by $O(1 /(x|\log x|))$ and $O(\log x)$ if $t=0$. They show that $\psi$, which is initially defined only in the upper half plane, actually has an analytic continuation to all of $\mathbb{C}$ apart from the negative real axis.

To each period function is also associated a periodic and holomorphic function $f$ on the upper half plane,

$$
f(z)=\psi(z)+z^{-2 s} \psi(-1 / z) .
$$

MSC2010: primary 11M06; secondary 11M41, 11L99.

Keywords: period functions, moments, mean values, Riemann zeta function, Eisenstein series,

Voronoi formula, cotangent sums, Vasyunin sum, Dedekind sum. 
In this paper we focus on the case of real analytic Eisenstein series. For these, the periodic function $f$ turns out to be essentially

$$
\sum_{n=1}^{\infty} \sigma_{2 s-1}(n) \mathrm{e}(n z)
$$

where, as usual, $\sigma_{a}(n):=\sum_{d \mid n} d^{a}$ indicates the sum of the $a$-th power of the divisors of $n$ and $\mathrm{e}(z):=e^{2 \pi i z}$. We interpret Lewis and Zagier's results directly in terms of this function, obtaining a better understanding of the Taylor series of the associated period function. It turns out that the case $s=1 / 2$, that is, $t=0$, is especially useful. In this case the arithmetic part of the $n$-th Fourier coefficient is $d(n)$, the number of divisors of $n$.

There are several nice applications that are consequences of the analytic continuation of the associated period function, that is, they are consequences of the surprising fact that the function

$$
\sum_{n=1}^{\infty} d(n) e(n z)-\frac{1}{z} \sum_{n=1}^{\infty} d(n) e(-n / z),
$$

which apparently only makes sense when the imaginary part of $z$ is positive, actually has an analytic continuation to $\mathbb{C}^{\prime}$ the slit complex plane (the complex with the negative real axis removed). First, we obtain a new formula for the weighted mean square of the Riemann zeta function on the critical line:

$$
\int_{0}^{\infty}|\zeta(1 / 2+i t)|^{2} e^{-\delta t} d t
$$

Previously, the best formula for this quantity was a main term plus an asymptotic, but not convergent, series of powers of $\delta$, each term an order of magnitude better than the previous as $\delta \rightarrow 0^{+}$. Our formula gives an asymptotic series that is also convergent. The situation is somewhat analogous to the situation of the partition function $p(n)$. Hardy and Ramanujan found an asymptotic series for $p(n)$ and subsequently Rademacher gave a series that was both asymptotic and convergent. In both the partition case and our case, the exact formula allows for the computation of the sought quantity to any desired degree of precision, whereas an asymptotic series has limits to its precision. Of course, an extra feature of $p(n)$, which is not present in our situation, is that since $p(n)$ is an integer it is known exactly once it is known to a precision of 0.5 . However, our formula does have the extra surprising feature that the time required to calculate our desired mean square is basically independent of $\delta$, apart from the intrinsic difficulty of the extra work required just to write down a high precision number $\delta$.

A second application proves a surprising reciprocity formula for the Vasyunin sum, which is a cotangent sum that appears in the Nyman-Beurling criterion for the 
Riemann hypothesis. Specifically, the Vasyunin sum appears as part of the exact formula for the twisted mean-square of the Riemann zeta function on the critical line:

$$
\int_{0}^{\infty}|\zeta(1 / 2+i t)|^{2}(h / k)^{i t} \frac{d t}{\frac{1}{4}+t^{2}}
$$

The fact that there is a reciprocity formula for the Vasyunin sum is a nonobvious symmetry relating this integral for $h / k$ and the integral for $\bar{h} / k$ where $h \bar{h} \equiv 1$ $\bmod k$. It is not apparent from this integral that there should be such a relationship; our formula reveals a hidden structure.

The reciprocity formula is most simply stated in terms of the function

$$
c_{0}(h / k)=-\sum_{m=1}^{k-1} \frac{m}{k} \cot \frac{\pi m h}{k},
$$

defined initially for nonzero rational numbers $h / k$ where $h$ and $k$ are integers with $(h, k)=1$ and $k>0$. The reciprocity formula can be simply stated as, "The function

$$
c_{0}\left(\frac{h}{k}\right)+\frac{k}{h} c_{0}\left(\frac{k}{h}\right)-\frac{1}{\pi h}
$$

extends from its initial definition on rationals $x=h / k$ to an (explicit) analytic function on the complex plane with the negative real axis deleted." This is nearly an example of what Zagier calls a "quantum modular form" [Zagier 2010]. We proved this reciprocity formula in [Bettin and Conrey 2011]; in this paper, we generalize it to a family of "cotangent sums", containing both $c_{0}$ and the Dedekind sum.

These (imperfect) quantum modular forms are analogous to the "quantum Maass forms" studied by Bruggeman [2007], the former being associated to Eisenstein series and the latter to Maass forms. The main difference between these two classes of quantum forms comes from the fact that the $L$-functions associated to Maass forms are entire, while for Eisenstein series the associated $L$-functions are not, since they are products of two shifted Riemann zeta functions. This translates into quantum Maass forms being quantum modular forms in the strict sense, whereas the reciprocity formulas for the cotangent sums contain a nonsmooth correction term.

As a third application, we give a generalization of the classical Voronoi summation formula, which is a formula for $\sum_{n=1}^{\infty} d(n) f(n)$, where $f(n)$ is a smooth rapidly decaying function. The usual formula proceeds from

$$
\sum_{n=1}^{\infty} d(n) f(n)=\frac{1}{2 \pi i} \int_{(2)} \zeta(s)^{2} \tilde{f}(s) d s, \quad \text { where } \tilde{f}(s)=\int_{0}^{\infty} f(x) x^{-s} d x .
$$


One obtains the formula by moving the path of integration to the left to $\operatorname{Re} s=-1$, say, and then using the functional equation

$$
\zeta(s)=\chi(s) \zeta(1-s)
$$

of $\zeta(s)$. Here, as usual,

$$
\chi(s)=2(2 \pi)^{s-1} \Gamma(1-s) .
$$

In this way one obtains a leading term

$$
\int_{0}^{\infty} f(u)(\log u+2 \gamma) d u
$$

from the pole of $\zeta(s)$ at $s=1$, plus another term

$$
\sum_{n=1}^{\infty} d(n) \hat{f}(n)
$$

where $\hat{f}(u)$ is a kind of Fourier-Bessel transform of $f$; specifically,

$$
\hat{f}(u)=\frac{1}{2 \pi i} \int_{(-1)} \chi(s)^{2} u^{s-1} \tilde{f}(s) d s=\int_{0}^{\infty} f(t) C(2 \pi \sqrt{t u}) d t
$$

with $C(z)=4 K_{0}(2 z)-2 \pi Y_{0}(2 z)$, where $K$ and $Y$ are the usual Bessel functions. By contrast, the period relation implies, for example, that for $0<\delta<\pi$ and $z=1-e^{-i \delta}$,

$$
\sum_{n=1}^{\infty} d(n) \mathrm{e}(n z)=\frac{1}{4}+2 \frac{\log (-2 \pi i z)-\gamma}{2 \pi i z}+\frac{1}{z} \sum_{n=1}^{\infty} d(n) \mathrm{e}\left(\frac{-n}{z}\right)+\sum_{n=1}^{\infty} c_{n} e^{-i n \delta},
$$

where $c_{n} \ll e^{-2 \sqrt{\pi n}}$. This is a useful formula that cannot be readily extracted from the Voronoi formula. In fact, the Voronoi formula is actually an easy consequence of the formula (2). In Section 4 we give some other applications of this extended Voronoi formula.

The theory and applications described above are for the period function associated with the Eisenstein series with $s=1 / 2$. In this paper we work in a slightly more general setting with $s=a$, an arbitrary complex number. The circle of ideas presented here have other applications and further generalizations, for example to exact formulas for averages of Dirichlet $L$-functions, which will be explored in future work. 


\section{Statement of results}

For $a \in \mathbb{C}$ and $\operatorname{Im}(z)>0$, consider

$$
\mathscr{S}_{a}(z):=\sum_{n=1}^{\infty} \sigma_{a}(n) \mathrm{e}(n z) .
$$

For $a=2 k+1$ with $k \in \mathbb{Z}_{\geq 1}$, the series $\mathscr{S}_{a}(z)$ is essentially the Eisenstein series of weight $2 k+2$ :

$$
E_{a+1}(z)=1+\frac{2}{\zeta(-a)} \mathscr{P}_{a}(z),
$$

for which the well-known modularity property

$$
E_{2 k}(z)-\frac{1}{z^{2 k}} E_{2 k}\left(-\frac{1}{z}\right)=0
$$

holds when $k \geq 2$. For other values of $a$ this equality is no longer true, but the period function

$$
\psi_{a}(z):=E_{a+1}(z)-\frac{1}{z^{a+1}} E_{a+1}\left(-\frac{1}{z}\right)
$$

still has some remarkable properties.

Theorem 1. Let $\operatorname{Im}(z)>0$ and $a \in \mathbb{C}$. Then $\psi_{a}(z)$ satisfies the three-term relation

$$
\psi_{a}(z)-\psi_{a}(z+1)=\frac{1}{(z+1)^{1+a}} \psi_{a}\left(\frac{z}{z+1}\right)
$$

and extends to an analytic function on $\mathbb{C}^{\prime}:=\mathbb{C} \backslash \mathbb{R}_{\leq 0}$ via the representation

$$
\psi_{a}(z)=\frac{i}{\pi z} \frac{\zeta(1-a)}{\zeta(-a)}-i \frac{1}{z^{1+a}} \cot \frac{\pi a}{2}+i \frac{g_{a}(z)}{\zeta(-a)},
$$

where

$$
\begin{aligned}
g_{a}(z):=-2 \sum_{1 \leq n \leq M}(-1)^{n} \frac{B_{2 n}}{(2 n) !} \zeta(1-2 n-a)(2 \pi z)^{2 n-1} \\
+\frac{1}{\pi i} \int_{\left(-\frac{1}{2}-2 M\right)} \zeta(s) \zeta(s-a) \Gamma(s) \frac{\cos \pi a / 2}{\sin \pi(s-a) / 2}(2 \pi z)^{-s} d s,
\end{aligned}
$$

and $M$ is any integer greater or equal to $-\frac{1}{2} \min (0, \operatorname{Re}(a))$.

Here and throughout the paper equalities are to be interpreted as identities between meromorphic functions in $a$. In particular, taking the limit $a \rightarrow 0^{+}$, we have

$$
\begin{aligned}
& \psi_{0}(z)=-2 \frac{\log 2 \pi z-\gamma}{\pi i z}-2 i g_{0}(z), \\
& g_{0}(z)=\frac{1}{\pi i} \int_{\left(-\frac{1}{2}\right)} \zeta(s)^{2} \frac{\Gamma(s)}{\sin (\pi s / 2)}(2 \pi z)^{-s} d s=\frac{1}{\pi i} \int_{\left(-\frac{1}{2}\right)} \frac{\zeta(s) \zeta(1-s)}{\sin \pi s} z^{-s} d s .
\end{aligned}
$$


Theorem 1 is essentially a reformulation of Lewis and Zagier's results [2001] for the noncuspidal case and can be seen as a starting point for their theory of period functions.

For ease of reference, note that (3) can be rewritten in terms of $\mathscr{Y}_{a}$ and $g_{a}$ as

$$
\begin{aligned}
\mathscr{S}_{a}(z)-\frac{1}{z^{a+1}} & \mathscr{S}_{a}\left(-\frac{1}{z}\right) \\
& =i \frac{\zeta(1-a)}{2 \pi z}-\frac{\zeta(-a)}{2}+\frac{e^{\pi i(a+1) / 2} \zeta(a+1) \Gamma(a+1)}{(2 \pi z)^{a+1}}+\frac{i}{2} g_{a}(z) .
\end{aligned}
$$

Another important feature of the function $\psi_{a}(z)$ comes from the properties of its Taylor series. For example, in the case $a=0$ one has

$$
\frac{\pi i}{2}(1+z) \psi_{0}(1+z)=-1-\frac{z}{2}+\sum_{m=2}^{\infty} a_{m}(-z)^{m},
$$

with

$$
a_{m}:=\frac{1}{n(n+1)}+2 b_{n}+2 \sum_{j=0}^{n-2}\left(\begin{array}{c}
n-1 \\
j
\end{array}\right) b_{j+2} \quad \text { and } \quad b_{n}:=\frac{\zeta(n) B_{n}}{n}
$$

and where $B_{2 n}$ denotes the $2 n$-th Bernoulli number. In particular, the values $a_{m}$ are rational polynomials in $\pi^{2}$. The terms involved in the definition of $a_{m}$ are extremely large, since

$$
b_{2 n} \sim \frac{B_{2 n}}{2 n} \sim(-1)^{n+1} 2 \sqrt{\frac{\pi}{n}}\left(\frac{n}{\pi e}\right)^{2 n}
$$

as $n \rightarrow \infty$, though there is a lot of cancellation; for example, for $m=20$ one has

$$
\begin{aligned}
a_{m}=\frac{1}{420}+\frac{\pi^{2}}{36}-\frac{19 \pi^{4}}{600}+\frac{646 \pi^{6}}{19845}-\frac{323 \pi^{8}}{1500}+\frac{4199 \pi^{10}}{343035} \\
-\frac{154226363 \pi^{12}}{36569373750}+\frac{1292 \pi^{14}}{1403325}-\frac{248571091 \pi^{16}}{2170943775000} \\
\quad+\frac{1924313689 \pi^{18}}{288905366499750}-\frac{30489001321 \pi^{20}}{252669361772953125} \\
=0.0499998087 \ldots
\end{aligned}
$$

Notice how close this number is to $\frac{1}{20}$; this observation can be made for all $m$ and in fact in [Bettin and Conrey 2011] we proved that

$$
a_{m}-\frac{1}{m}=2^{5 / 4} \pi^{3 / 4} \frac{e^{-2 \sqrt{\pi m}}}{m^{3 / 4}}\left(\sin \left(2 \sqrt{\pi m}+\frac{3}{8} \pi\right)+O\left(\frac{1}{\sqrt{m}}\right)\right) .
$$


In this paper we show that similar results hold for the Taylor series at any point $\tau$ in the half plane $\operatorname{Re}(\tau)>0$ and for any $a \in \mathbb{C}$. We give a proof in the following theorem, using $g_{a}$ instead of $\psi_{a}$ to simplify slightly the resulting formulas.

Theorem 2. Let $\operatorname{Re}(\tau)>0$ and for $|z|<|\tau|$, let

$$
g_{a}(\tau+z):=\sum_{m=0}^{\infty} \frac{g_{a}^{(m)}(\tau)}{m !} z^{m}
$$

be the Taylor series of $g_{a}(z)$ around $\tau$. Then

$$
\begin{array}{r}
\frac{g_{a}^{(m)}(1)}{m !}=-\sum_{\substack{2 n-1+k=m, n, k \geq 1}}(-1)^{n+m} B_{2 n} \zeta \\
+(-1)^{m} \cot \frac{\pi a}{2} \zeta(-a) \frac{\Gamma(1+a+m)}{\Gamma(1+a) m !} \\
+(-1)^{m}\left(\frac{\Gamma(1+a+m)}{\Gamma(a)(m+1) !}-1\right) \frac{\zeta(1-a)}{\pi}
\end{array}
$$

and in particular if $a \in \mathbb{Z}_{\leq 0}$ and $(a, m) \neq(0,0)$, then $\pi g_{a}^{(m)}(1)$ is a rational polynomial in $\pi^{2}$. Moreover,

$$
\begin{aligned}
\frac{g_{a}^{(m)}(\tau)}{m !}=\cos & \left.\frac{\pi a}{2}\right) \frac{2^{7 / 4-a / 2}}{\pi^{3 / 4+a / 2}} \frac{e^{-2 \sqrt{\pi \tau m}}}{m^{1 / 4-a / 2} \tau^{m+3 / 4+a / 2}} \\
& \times\left(\cos \left(2 \sqrt{\pi \tau m}-\frac{1}{8} \pi(2 a-1)+(\tau+m) \pi\right)+O_{\tau, a}\left(\frac{1}{\sqrt{m}}\right)\right),
\end{aligned}
$$

as $m \rightarrow \infty$.

Some of the ideas used in the proofs of Theorems 1 and 2 can be easily generalized to a more general setting. For example, let $F(s)$ be a meromorphic function on $1-\omega \leq \operatorname{Re}(s) \leq \omega$ for some $1<\omega<2$ with no poles on the boundary and assume $|F(\sigma+i t)| \ll_{\sigma} e^{(\pi / 2-\eta)|t|}$ for some $\eta>0$. Let

$$
\begin{aligned}
& W_{+}(z):=\frac{1}{2 \pi i} \int_{(\omega)} F(s) \Gamma(s)(-2 \pi i z)^{-s} d s, \\
& W_{-}(z):=\frac{1}{2 \pi i} \int_{(\omega)} F(1-s) \Gamma(s)(-2 \pi i z)^{-s} d s,
\end{aligned}
$$

for $\frac{\pi}{2}-\eta<\arg z<\frac{\pi}{2}+\eta$. (Notice that these functions are essentially convolutions of the exponential function and the Mellin transform of $F(s)$.) Then we have

$$
\sum_{n=1}^{\infty} d(n) W_{+}(n z)-\frac{1}{z} \sum_{n=1}^{\infty} d(n) W_{-}\left(-\frac{n}{z}\right)=R(z)+k(z),
$$


where $R(z)$ is the sum of the residues of $F(s) \Gamma(s) \zeta(s)^{2}(-2 \pi i z)^{-s}$ between $1-\omega$ and $\omega$, and

$$
k(z):=\frac{1}{2 \pi} \int_{(1-\omega)} F(s) \frac{\zeta(s) \zeta(1-s)}{\sin \pi s} z^{-s} d s
$$

is holomorphic on $|\arg (z)|<\frac{\pi}{2}+\eta$. Moreover, if we assume that $F(s)$ is holomorphic on $\operatorname{Re}(s)<1-\omega$, then it follows that the Taylor series of $k(z)$ converges very fast, that is,

$$
\frac{k^{(n)}(\tau)}{n !} \ll n^{-B}|\tau|^{-n}
$$

for any $B>0$ and $\tau$ such that $|\arg \tau|<\eta$. Also, $W_{-}(z)$ decays faster than any power of $z$ at infinity and so the second sum in (10) is rapidly convergent and is very small if we let $z$ go to zero in $|\arg z|<\eta$. In Section 4 we will give an explicit example; a subsequent paper will elaborate on this.

The Voronoi summation formula is an important tool in analytic number theory; in its simplest form, it states that, if $f(u)$ is a smooth function of compact support, then

$$
\sum_{n=1}^{\infty} d(n) f(n)=\sum_{n=1}^{\infty} d(n) \hat{f}(n)+\int_{0}^{\infty} f(t)(\log t+2 \gamma) d t+\frac{1}{4} f(0),
$$

where

$$
\hat{f}(x):=4 \int_{0}^{\infty} f(t)\left(K_{0}(4 \pi \sqrt{t x})-\frac{1}{2} \pi Y_{0}(4 \pi \sqrt{t x})\right) d t .
$$

This formula can be deduced from (10) (or also directly from (6)) as a very easy corollary. Actually, Voronoi's formula can be interpreted as a version of (6) confined to the positive real axis. If we get rid of this limitation and we use directly the period formula (6), we are able to obtain interesting results also for weight functions of the shape $f(u)=e^{-\delta u}$, for which the Voronoi summation formula fails to give a useful formula. (Try it!) Thus, we have a generalization of Voronoi's formula.

The use of a weight function of the shape $e^{-\delta u}$ is fundamental to investigate the smoothly weighted second moment of the Riemann zeta function,

$$
L_{2 k}(\delta):=\int_{0}^{\infty}\left|\zeta\left(\frac{1}{2}+i t\right)\right|^{2 k} e^{-\delta t} d t,
$$

in the case $k=1$. These integrals play a major role in the theory of the Riemann zeta function and getting good upper bounds on their growth as $\delta \rightarrow 0^{+}$would imply the Lindelöf hypothesis. Unfortunately, the only two value of $k$ for which the asymptotics are known are $k=1$ [Hardy and Littlewood 1916] and $k=2$ [Ingham 1927]. For other values we have just conjectures; see [Conrey and Ghosh 1998; Conrey and Gonek 2001; Keating and Snaith 2000]. For $k=1$, it is easy to 
see that the smooth moment is strictly related to the sum $\mathscr{Y}_{0}\left(-e^{-i \delta}\right)$ and, from this, it is easy to deduce an asymptotic expansion for $L_{2 k}(\delta)$. This classical asymptotic series is not convergent. Here we replace the series by two series, each of which are absolutely convergent asymptotic series. (See also [Motohashi 1997].) The following theorem provides a new exact formula for $L_{1}(\delta)$, by applying Theorem 1 and 2 to $\mathscr{Y}_{0}\left(-e^{-i \delta}\right)$.

Theorem 3. For $0<\operatorname{Re}(\delta)<\pi$, we have

$$
L_{1}(\delta)=\frac{\gamma-\log 2 \pi \delta}{2 \sin \delta / 2}+\frac{\pi i}{\sin \delta / 2} \mathscr{Y}_{0}\left(\frac{-1}{1-e^{-i \delta}}\right)+h(\delta)+k(\delta),
$$

where $k(\delta)$ is analytic in $|\operatorname{Re}(\delta)|<\pi$ and $h(\delta)$ is $C^{\infty}$ in $\mathbb{R}$ and holomorphic in

$$
\mathbb{C}^{\prime \prime}:=\mathbb{C} \backslash\{x+i y \in \mathbb{C} \mid x \in 2 \pi \mathbb{Z}, y \geq 0\} .
$$

Moreover, $h(0)=0$ and, if $\operatorname{Im}(\delta) \leq 0$,

$$
h(\delta)=i \sum_{n \geq 0} h_{n} e^{-i(n+1 / 2) \delta},
$$

with

$$
h_{n}=2^{7 / 4} \pi^{1 / 4} \frac{e^{-2 \sqrt{\pi n}}}{n^{1 / 4}} \sin \left(2 \sqrt{\pi n}+\frac{5}{8} \pi\right)+O\left(\frac{e^{-2 \sqrt{\pi n}}}{n^{3 / 4}}\right),
$$

as $n \rightarrow \infty$.

The most remarkable aspect of this theorem lies in the fact that the arithmetic sum $\mathscr{S}_{0}\left(-1 /\left(1-e^{-i \delta}\right)\right)$ decays exponentially fast for $\delta \rightarrow 0^{+}$, while the Fourier series $h(\delta)$ is very rapidly convergent. Moreover, Theorem 3 implies that $L_{1}(\delta)$ can be evaluated to any given precision in a time that is independent of $\delta$.

For a rational number $h / k$, with $(h, k)=1$ and $k>0$, define

$$
c_{0}\left(\frac{h}{k}\right)=-\sum_{m=1}^{k-1} \frac{m}{k} \cot \left(\frac{\pi m h}{k}\right) .
$$

The value of $c_{0}(h / k)$ is an algebraic number, that is, $c: \mathbb{Q} \rightarrow \overline{\mathbb{Q}}$, and, more precisely, $c_{\ell}(h / k)$ is contained in the maximal real subfield of the cyclotomic field of $k$-th roots of unity. Moreover, $c_{0}$ is odd and is periodic of period 1. See Figure 1 and Figure 2.

The cotangent sum $c_{0}(h / k)$ arises in analytic number theory in the value

$$
D(0, h / k)=\frac{1}{4}+\frac{i}{2} c_{0}\left(\frac{h}{k}\right)
$$




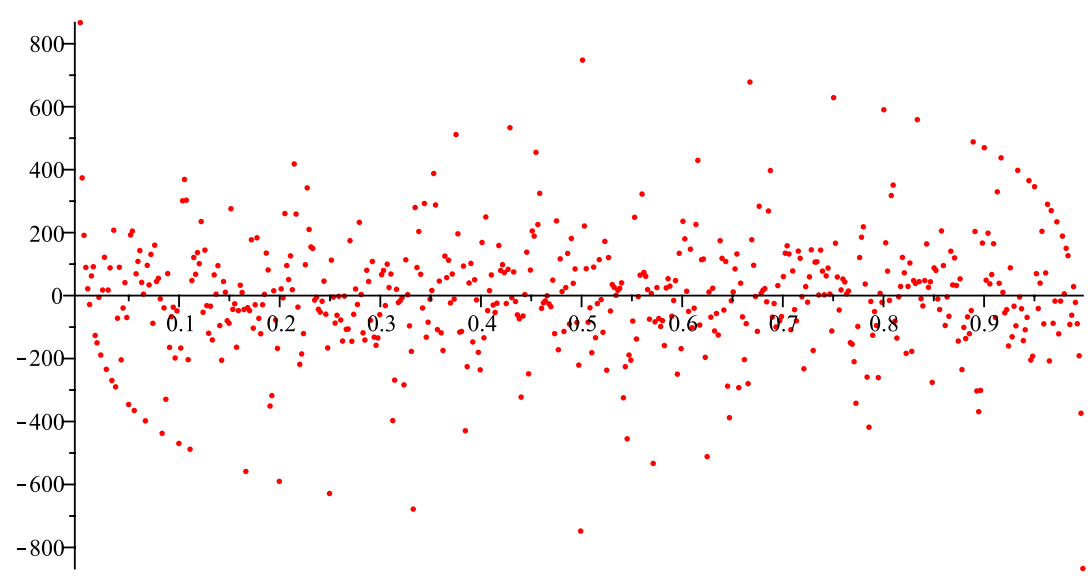

Figure 1. Graph of $c_{0}(h / k)$ for $1 \leq h<k=541$.

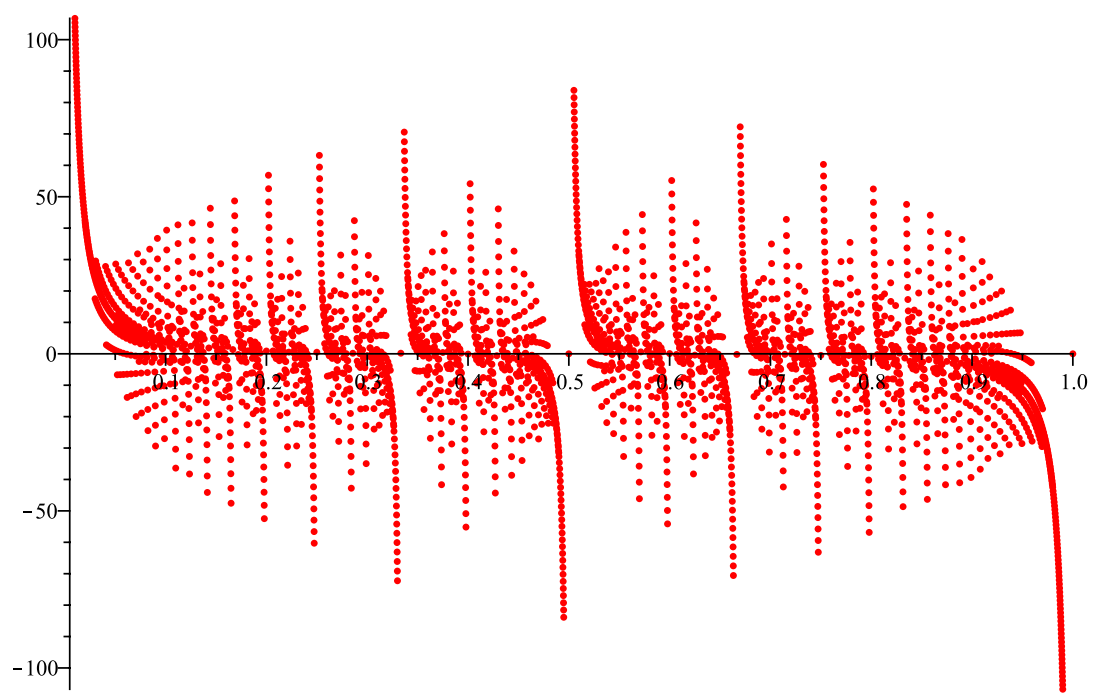

Figure 2. Graph of $c_{0}(h / k)$ for $1 \leq h \leq k \leq 100$, with $(h, k)=1$.

at $s=0$ of the Estermann function, defined for $\operatorname{Re}(s)>1$ by

$$
D(s, h / k):=\sum_{n=1}^{\infty} \frac{d(n) \mathrm{e}(n h / k)}{n^{s}} .
$$

The Estermann function extends analytically to $\mathbb{C} \backslash\{1\}$ and satisfies a functional equation; these properties are useful in studying the asymptotics of the mean square 


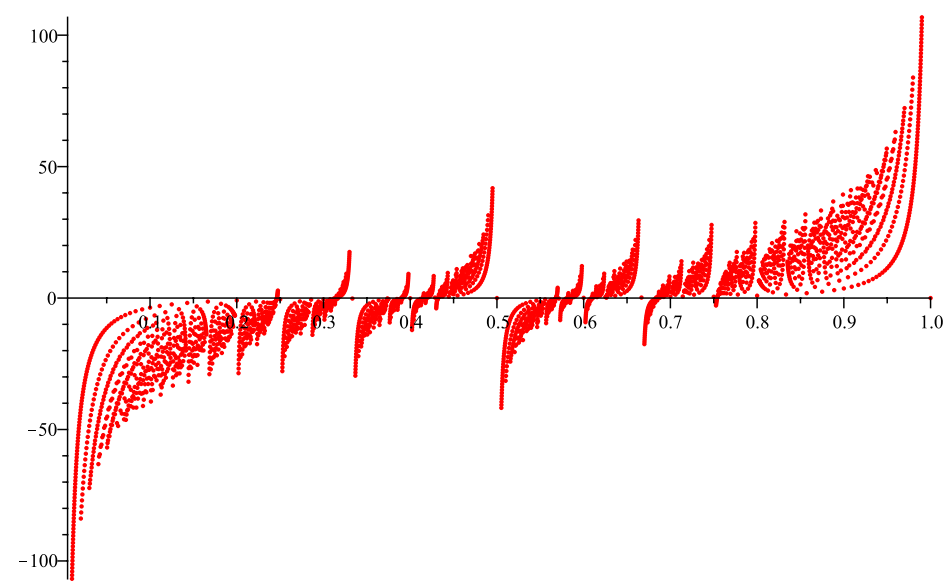

Figure 3. Graph of $V(h / k)$ for $1 \leq h, k \leq 100$ and $(h, k)=1$.

of the Riemann zeta function multiplied by a Dirichlet polynomial (see [Balasubramanian et al. 1985]), which are needed, for example, for theorems that give a lower bound for the portion of zeros of $\zeta(s)$ on the critical line. See also [Conrey 1989; Iwaniec 1980]. The sum

$$
V\left(\frac{h}{k}\right):=\sum_{m=1}^{k-1}\left\{\frac{m h}{k}\right\} \cot \left(\frac{\pi m}{k}\right)=-c_{0}(\bar{h} / k),
$$

known as the Vasyunin sum (see Figure 3), arises in the study of the Riemann zeta function by virtue of the formula

$$
\begin{aligned}
v(h / k) & :=\frac{1}{2 \pi \sqrt{h k}} \int_{-\infty}^{\infty}\left|\zeta\left(\frac{1}{2}+i t\right)\right|^{2}\left(\frac{h}{k}\right)^{i t} \frac{d t}{\frac{1}{4}+t^{2}} \\
& =\frac{\log 2 \pi-\gamma}{2}\left(\frac{1}{h}+\frac{1}{k}\right)+\frac{k-h}{2 h k} \log \frac{h}{k}-\frac{\pi}{2 h k}\left(V\left(\frac{h}{k}\right)+V\left(\frac{k}{h}\right)\right) ;
\end{aligned}
$$

see Figure 4.

This formula is relevant to the approach of Nyman, Beurling, Báez-Duarte and Vasyunin to the Riemann hypothesis, which asserts that the Riemann hypothesis is true if and only if $\lim _{N \rightarrow \infty} d_{N}=0$, where

$$
d_{N}^{2}=\inf _{A_{N}} \frac{1}{2 \pi} \int_{-\infty}^{\infty}\left|1-\zeta A_{N}\left(\frac{1}{2}+i t\right)\right|^{2} \frac{d t}{\frac{1}{4}+t^{2}}
$$

and the infimum is over all the Dirichlet polynomial $A_{N}(s)=\sum_{n=1}^{N} a_{n} / n^{s}$ of length $N$; see [Bagchi 2006] for a nice account of the Nyman-Beurling approach to the 


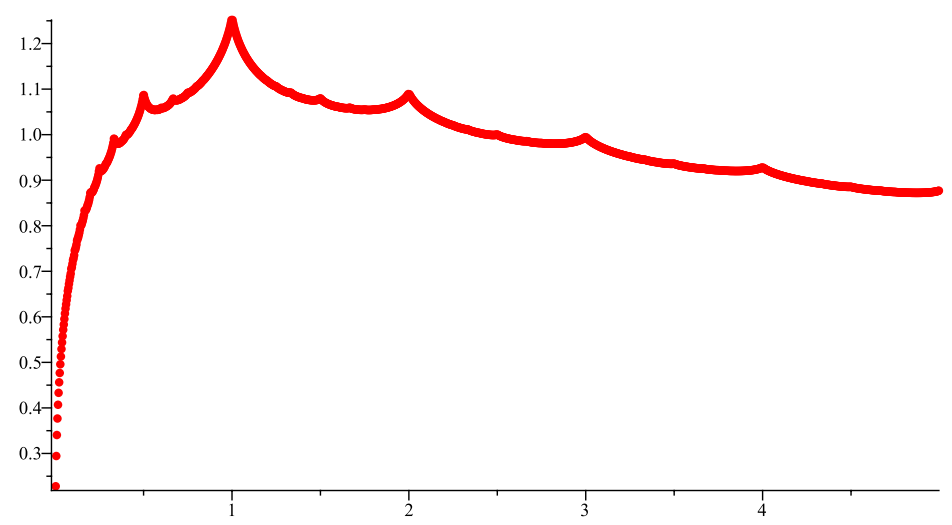

Figure 4. Graph of $\sqrt{h k} v(h / k)$ for $1 \leq h \leq 5 k, k=307$, and $(h, k)=1$.

Riemann hypothesis with Báez-Duarte's significant contribution and see [BáezDuarte et al. 2005; Landreau and Richard 2002] for information about the Vasyunin sums, as well as interesting numerical experiments about $d_{N}$ and the minimizing polynomials $A_{N}$. Thus $d_{N}^{2}$ is a quadratic expression in the unknown quantities $a_{m}$ in terms of the Vasyunin sums.

In [Bettin and Conrey 2011] we showed that $c_{0}(h / k)$ satisfies the reciprocity formula

$$
c_{0}\left(\frac{h}{k}\right)+\frac{k}{h} c_{0}\left(\frac{k}{h}\right)-\frac{1}{\pi h}=\frac{i}{2} \psi_{0}\left(\frac{h}{k}\right)
$$

(and in particular that $c_{0}(h / k)$ can be computed to within a prescribed accuracy in a time that is polynomial in $\log k$ ). See Figure 5.

This behavior is analogous to that of the Dedekind sum,

$$
s\left(\frac{h}{k}\right)=-\frac{1}{4 k} \sum_{m=1}^{k-1} \cot \left(\frac{\pi m}{k}\right) \cot \left(\frac{\pi m h}{k}\right),
$$

which satisfies the well-known reciprocity formula

$$
s\left(\frac{h}{k}\right)+s\left(\frac{k}{h}\right)-\frac{1}{12 h k}=\frac{1}{12}\left(\frac{h}{k}+\frac{k}{h}-3\right) .
$$

In this paper we prove that these results can be generalized to the sums

$$
c_{a}\left(\frac{h}{k}\right):=k^{a} \sum_{m=1}^{k-1} \cot \left(\frac{\pi m h}{k}\right) \zeta\left(-a, \frac{m}{k}\right),
$$

where $\zeta(s, x)$ is the Hurwitz zeta function (note that at $a=-1$ the poles of $\zeta(-a, m / k)$ cancel $)$. 


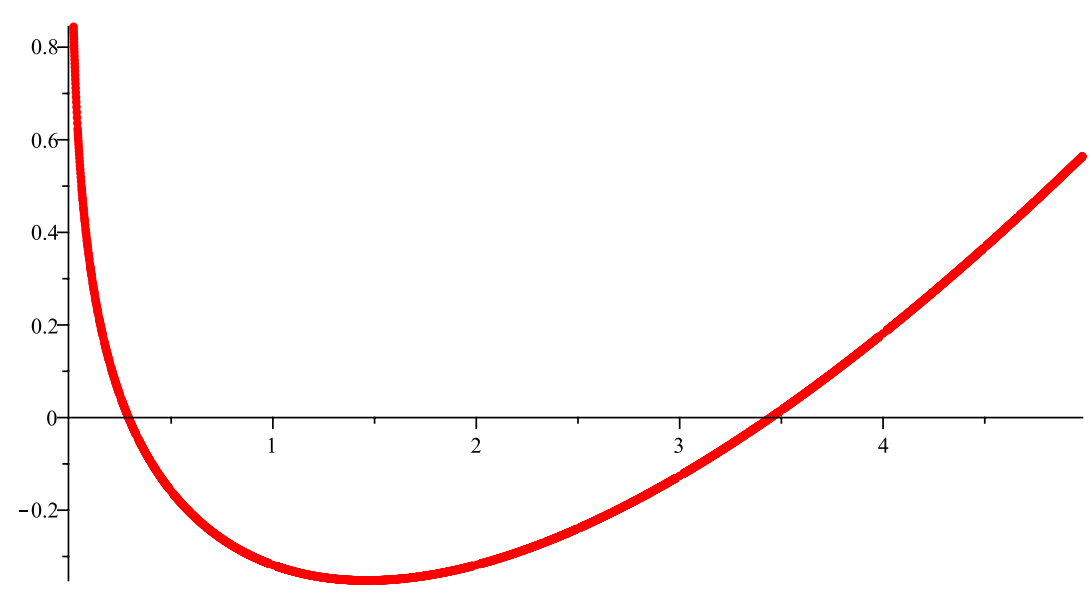

Figure 5. Graph of $c_{0}(h / k)+(k / h) c_{0}(k / h)-1 / \pi h$ for $h \leq 5 k$, $k \leq 50$ and $(h, k)=1$.

Notice that, for all $a, c_{a}(h / k)$ is odd and periodic in $x=h / k$ with period 1 and, for nonnegative integers $a$, it takes values in the maximal real subfield of the cyclotomic field of $k$-th roots of unity.

At the nonnegative integers, $a=n \geq 0$, these cotangent sums can be expressed in terms of the Bernoulli polynomials:

$$
c_{n}\left(\frac{h}{k}\right)=-k^{n} \sum_{m=1}^{k-1} \cot \left(\frac{\pi m h}{k}\right) \frac{B_{n+1}(m / k)}{n+1},
$$

which is most interesting when $n$ is even, since $c_{n} \equiv 0$ for positive odd $n$.

If $a=-n$ is a negative integer one can write $c_{a}$ as

$$
c_{-n}\left(\frac{h}{k}\right)=\frac{(-1)^{n}}{k^{n}(n-1) !} \sum_{m=1}^{k-1} \cot \left(\frac{\pi m h}{k}\right) \Psi\left(n-1, \frac{m}{k}\right),
$$

where

$$
\Psi(m, z):=\frac{d^{m+1}}{d z^{m+1}} \log \Gamma(z)
$$

is the polygamma function.

By the reflection formula for the polygamma function,

$$
\Psi(m, 1-z)+(-1)^{m+1} \Psi(m, z)=(-1)^{m} \pi \frac{d^{m}}{d z^{m}} \cot (\pi z),
$$


for a positive odd integer $n$ we can write $c_{-n}$ as

$$
c_{-n}\left(\frac{h}{k}\right)=-\left.\frac{\pi}{2 k^{n}(n-1) !} \sum_{m=1}^{k-1} \cot \left(\frac{\pi m h}{k}\right) \frac{d^{n-1}}{d z^{n-1}} \cot (\pi z)\right|_{z=m / k}
$$

and, in particular,

$$
c_{-1}\left(\frac{h}{k}\right)=2 \pi s\left(\frac{h}{k}\right) .
$$

Like the case $a=0$, these cotangent sums appear in the value

$$
D\left(0, a, \frac{h}{k}\right)=-\frac{1}{2} \zeta(-a)+\frac{i}{2} c_{a}\left(\frac{h}{k}\right),
$$

at $s=0$ of the function $D(s, a, h / k)$, defined for $\operatorname{Re}(s)>1$ by

$$
D\left(s, a, \frac{h}{k}\right):=\sum_{n=1}^{\infty} \frac{\sigma_{a}(n) \mathrm{e}(n h / k)}{n^{s}} .
$$

Moreover, the cotangent sums $c_{a}$ appear also in a shifted version of Vasyunin's formula (13) (see Theorem 5 at the end of the paper for a new analytic proof).

Theorem 4. Let $h, k \geq 1$, with $(h, k)=1$. Then

$$
c_{a}\left(\frac{h}{k}\right)-\left(\frac{k}{h}\right)^{1+a} c_{a}\left(\frac{-k}{h}\right)+k^{a} \frac{a \zeta(1-a)}{\pi h}=-i \zeta(-a) \psi_{a}\left(\frac{h}{k}\right) .
$$

(Note that, since $g_{-1}(z)$ is identically zero, for $a=-1$ the reciprocity formula reduces to (15).) In particular, $c_{a}(h / k)$ gives an example of an "imperfect" quantum modular form of weight $1+a$.

New formulas can be obtained by differentiating (17); for example, if we write

$$
c_{-1}^{*}\left(\frac{h}{k}\right):=\frac{1}{k} \sum_{m=1}^{k-1} \cot \left(\frac{\pi m h}{k}\right) \gamma_{1}\left(\frac{m}{k}\right),
$$

where $\gamma_{1}(x)$ is the first generalized Stieltjes constant defined by

$$
\zeta(s, x)=\frac{1}{s-1}+\sum_{n=0}^{\infty} \frac{(-1)^{n}}{n !} \gamma_{n}(x)(s-1)^{n},
$$

then, taking the derivative at -1 of (17) multiplied by $k^{-a}$, we get the formula

$$
c_{-1}^{*}\left(\frac{h}{k}\right)-c_{-1}^{*}\left(\frac{-k}{h}\right)+\frac{\zeta^{\prime}(2)+\pi^{2} / 6}{\pi k h}+\pi \log k\left(\frac{1}{6} \frac{k}{h}-\frac{1}{2}\right)=q\left(\frac{h}{k}\right),
$$

where

$$
q(z):=-\frac{1}{\pi z} \zeta^{\prime}(2)+\frac{\pi}{2}(\log z+\gamma)+g_{-1}^{\prime}(z)
$$

is holomorphic in $\mathbb{C}^{\prime}$. 


\section{The period function}

In this section we give a proof of Theorems 1 and 2 .

Proof of Theorem 1. Firstly, observe that the three-term relation (4) follows easily from the periodicity in $z$ of $E(a, z)$.

$\mathscr{S}_{a}(z)$ can be written as

$$
\begin{aligned}
\mathscr{S}_{a}(z) & =\sum_{n=1}^{\infty} \sigma_{a}(n) \frac{1}{2 \pi i} \int_{(2+\max (0, \operatorname{Re}(a)))} \Gamma(s)(-2 \pi i n z)^{-s} d s \\
& =\frac{1}{2 \pi i} \int_{(2+\max (0, \operatorname{Re}(a)))} \zeta(s) \zeta(s-a) \Gamma(s) e^{\pi i s / 2}(2 \pi z)^{-s} d s \\
& =\frac{1}{2 \pi i} \int_{\left(-\frac{1}{2}-2 M\right)} \zeta(s) \zeta(s-a) \Gamma(s) e^{\pi i s / 2}(2 \pi z)^{-s} d s+r_{a, M}(z),
\end{aligned}
$$

where $M$ is any integer greater or equal to $-\frac{1}{2} \min (0, \operatorname{Re}(a))$ and

$$
\begin{aligned}
r_{a, M}(z):=-\frac{1}{2} \zeta(-a)+i \frac{\zeta(1-a)}{2 \pi z} & +i \frac{\zeta(1+a) \Gamma(1+a) e^{\pi i a / 2}}{(2 \pi z)^{1+a}} \\
& -\sum_{1 \leq n \leq M} i(-1)^{n} \frac{B_{2 n}}{(2 n) !} \zeta(1-2 n-a)(2 \pi z)^{2 n-1}
\end{aligned}
$$

is the sum of the residues encountered moving the integral (and has to be interpreted in the limit sense if some of the terms have a pole). Now, consider

$$
\begin{aligned}
\frac{1}{z^{1+a}} \mathscr{Y}_{a}\left(-\frac{1}{z}\right) & =\frac{1}{z^{1+a}} \frac{1}{2 \pi i} \int_{(2+\max (0, \operatorname{Re}(a)))} \zeta(s) \zeta(s-a) \Gamma(s) e^{\pi i s / 2}\left(2 \pi \frac{-1}{z}\right)^{-s} d s \\
& =\frac{1}{2 \pi i} \int_{(2+\max (0, \operatorname{Re}(a)))} \zeta(s) \zeta(s-a) \Gamma(s) e^{-\pi i s / 2}(2 \pi)^{-s} z^{s-1-a} d s,
\end{aligned}
$$

since in this context $0<\arg z<\pi$ and $0<\arg -1 / z<\pi$, so $\arg -1 / z=\pi-\arg z$. Applying the functional equation to both $\zeta(s)$ and $\zeta(s-a)$ we get, after the change of variable $s \rightarrow 1-s+a$,

$$
\begin{aligned}
\frac{1}{z^{1+a}} \varphi_{a}\left(-\frac{1}{z}\right) & \\
& =-\frac{1}{2 \pi} \int_{(-1+\min (0, \operatorname{Re}(a)))} \zeta(s-a) \zeta(s) \Gamma(s) \frac{e^{\pi i(s-a) / 2} \cos \frac{\pi s}{2}}{\sin \frac{\pi(s-a)}{2}}(2 \pi z)^{-s} d s \\
& =-\frac{1}{2 \pi} \int_{(-1 / 2-M)} \zeta(s-a) \zeta(s) \Gamma(s) \frac{e^{\pi i(s-a) / 2} \cos \frac{\pi s}{2}}{\sin \frac{\pi(s-a)}{2}}(2 \pi z)^{-s} d s,
\end{aligned}
$$


since the integrand doesn't have any pole on the left of $-1+\min (0, \operatorname{Re}(a))$. The theorem then follows summing (18) and (19) and using the identity

$$
e^{\pi i s / 2}+i \frac{e^{\pi i(s-a) / 2} \cos \frac{\pi s}{2}}{\sin \frac{\pi(s-a)}{2}}=i \frac{\cos \frac{\pi a}{2}}{\sin \frac{\pi(s-a)}{2}} .
$$

We remark that for $a=2 k+1$, with $k \geq 1$, Theorem 1 reduces to

$$
E_{2 k}(z)-\frac{1}{z^{2 k}} E_{2 k}\left(-\frac{1}{z}\right)=0,
$$

while, for $a=1$, the theorem reduces to the well-known identity

$$
E_{2}(z)-\frac{1}{z^{2}} E_{2}\left(\frac{-1}{z}\right)=-\frac{12}{2 \pi i z} .
$$

To prove Theorem 2 we need the following lemma.

Lemma 1. For fixed complex numbers $A$ and $\alpha$ we have, as $n \rightarrow \infty$

$$
\begin{aligned}
J_{n} & :=\int_{0}^{\infty} u^{n+\alpha} e^{-A \sqrt{u}} e^{-u} \frac{d u}{u} \\
& =\sqrt{2 \pi} e^{A^{2} / 8} e^{-A \sqrt{n}} e^{-n} n^{n+\alpha-1 / 2}\left(1-\frac{C}{\sqrt{n}}+O\left(\frac{1}{n}\right)\right),
\end{aligned}
$$

where

$$
C=\frac{4 \alpha-1}{8} A+\frac{A^{3}}{96} .
$$

Proof. After the change of variable $u=n x^{2}$, we have

$$
\begin{aligned}
& J_{n}= 2 n^{n+\alpha} \int_{0}^{\infty} x^{2 \alpha-1} e^{-A \sqrt{n} x-n\left(x^{2}-2 \log x\right)} d x \\
&= 2 n^{n+\alpha} e^{-A \sqrt{n}} \int_{-1}^{\infty}(x+1)^{2 \alpha-1} e^{-A \sqrt{n} x-n\left((x+1)^{2}-2 \log (x+1)\right)} d x \\
&=2 n^{n+\alpha} e^{-A \sqrt{n}} e^{-n}\left(1+O\left(e^{-n \delta^{2} / 2}\right)\right) \\
& \quad \quad \times \int_{-\delta}^{\delta}(x+1)^{2 \alpha-1} e^{-A \sqrt{n} x-2 n x^{2}}\left(1+\frac{2}{3} n x^{3}+O\left(n x^{4}\right)\right) d x
\end{aligned}
$$

for any small $\delta>0$. We can then approximate the binomial and extend the integral to $\mathbb{R}$ at a negligible cost, getting

$$
\begin{aligned}
& J_{n}=2 n^{n+\alpha} e^{-A \sqrt{n}} e^{-n} \int_{-\infty}^{\infty}\left(1+(2 \alpha-1) x+\frac{2}{3} n x^{3}+O\left(x^{2}+n x^{4}\right)\right) \\
& \times e^{-A \sqrt{n} x-2 n x^{2}} d x .
\end{aligned}
$$

Evaluating the integrals, the lemma follows. 
Proof of Theorem 2. The three-term relation (4) implies that

$$
\begin{aligned}
& g_{a}(z+1)=\frac{1}{(z+1)^{1+a}} \cot \left(\frac{\pi a}{2}\right) \zeta(-a)-\frac{1}{\pi z(z+1)^{a}} \zeta(1-a) \\
& \quad+\frac{1}{\pi z(z+1)} \zeta(1-a)+g_{a}(z)-\frac{1}{(z+1)^{1+a}} g_{a}\left(\frac{z}{z+1}\right)
\end{aligned}
$$

Now, from the definition (5) of $g_{a}(z)$, it follows that

$$
g_{a}(z)=2 \sum_{1 \leq n \leq M}(-1)^{n} \frac{B_{2 n}}{(2 n) !} \zeta(1-2 n-a)(2 \pi z)^{2 n-1}+O\left(|z|^{2 M+1 / 2}\right)
$$

for any $M \geq 1$. Thus

$$
\begin{array}{r}
g_{a}(z)-\frac{g_{a}(z /(z+1))}{(z+1)^{1+a}} \\
=2 \sum_{1 \leq n \leq M}(-1)^{n} \frac{B_{2 n}}{(2 n) !} \zeta(1-2 n-a)(2 \pi z)^{2 n-1}\left(1-\frac{1}{(z+1)^{2 n+a}}\right)+O\left(|z|^{2 M+1 / 2}\right) \\
=-2 \sum_{m=1}^{2 M}\left(\sum_{\substack{2 n-1+k=m, n, k \geq 1}}(-1)^{n+m} B_{2 n} \zeta(1-2 n-a) \frac{\Gamma(2 n+a+k)}{\Gamma(2 n+a) k !(2 n) !}(2 \pi)^{2 n-1}\right) z^{m} \\
+O\left(|z|^{2 M+\frac{1}{2}}\right) .
\end{array}
$$

Therefore,

$$
g_{a}(z+1)=\sum_{m=0}^{2 M} b_{m} z^{m}+O\left(|z|^{2 M+1 / 2}\right)
$$

where

$$
\begin{aligned}
b_{m}:=-2 \sum_{\substack{2 n-1+k=m, n, k \geq 1}}(-1)^{n+k} B_{2 n} \zeta(1-2 n-a) & \frac{\Gamma(2 n+a+k)}{\Gamma(2 n+a) k !(2 n) !}(2 \pi)^{2 n-1} \\
+(-1)^{m} \cot \left(\frac{\pi a}{2}\right) \zeta(-a) & \frac{\Gamma(1+a+m)}{\Gamma(1+a) m !} \\
& +(-1)^{m}\left(\frac{\Gamma(1+a+m)}{\Gamma(a)(m+1) !}-1\right) \frac{\zeta(1-a)}{\pi},
\end{aligned}
$$

and, since $g_{a}(z)$ is holomorphic at $1, b_{m}$ must coincide with the $m$-th coefficient of the Taylor series of $g_{a}(z)$ at 1 .

Now, let's prove the asymptotic (8). Fix any $M \geq-\frac{1}{2} \min (0, \operatorname{Re}(a))$ and assume $m \geq 2 M+1$ and $\operatorname{Re}(\tau)>0$. By the functional equation for $\zeta$ and basic properties 
of $\Gamma(s)$, we have

$$
\begin{aligned}
& \frac{(2 \pi)^{a} \tau^{m}}{\cos \frac{\pi a}{2}} g_{a}^{(m)}(\tau) \\
& =\frac{(-1)^{m}}{\pi i} \int_{\left(-\frac{1}{2}-2 M\right)} \Gamma(s) \frac{\zeta(s) \zeta(s-a)}{\sin \frac{\pi(s-a)}{2}} s(s+1) \cdots(s+m-1)(2 \pi)^{-s+a} \tau^{-s} d s \\
& =\frac{(-1)^{m}}{\pi i} \int_{\left(-\frac{1}{2}-2 M\right)} \frac{\zeta(s) \zeta(s-a)}{\sin \frac{\pi(s-a)}{2}} \Gamma(s+m)(2 \pi)^{-s+a} \tau^{-s} d s \\
& =\frac{(-1)^{m}}{\pi^{3} i} \int_{\left(-\frac{1}{2}-2 M\right)} \zeta(1-s) \zeta(1-s+a) \\
& \quad \times \Gamma(1-s) \Gamma(1-s+a) \Gamma(s+m) \sin \left(\frac{\pi s}{2}\right)\left(\frac{2 \pi}{\tau}\right)^{s} d s .
\end{aligned}
$$

We can see immediately that $g_{a}^{(m)}(\tau) \ll_{a} m^{-B}|\tau|^{-m} m$ ! for any fixed $B>0$, just by moving the path of integration to the line $\operatorname{Re}(s)=-B$ and using trivial estimates for $\Gamma$. To get a formula asymptotic as $m \rightarrow \infty$, we expand $\zeta(1-s) \zeta(1-s+a)$ into a Dirichlet series and integrate term-by-term; the main term arises from the first term of the sum. We have

$$
g_{a}^{(m)}(\tau)=2 \frac{(-\tau)^{-m} \cos \frac{\pi a}{2}}{\pi^{2}(2 \pi)^{a}} \sum_{\ell=1}^{\infty} \frac{\sigma_{-a}(\ell)}{\ell} I_{m, a}\left(\frac{\ell}{\tau}\right),
$$

where

$$
I_{m, a}(x):=\frac{1}{2 \pi i} \int_{\left(-\frac{1}{2}-2 M\right)} \Gamma(1-s) \Gamma(1-s+a) \Gamma(s+m) \sin \left(\frac{\pi s}{2}\right)(2 \pi x)^{s} d s .
$$

We reexpress this integral as a convolution integral. Recall that for $|\arg x|<\pi$ we have

$$
\frac{1}{2 \pi i} \int_{\left(\frac{3}{2}+2 M\right)} \Gamma(s) \Gamma(s+a) u^{-s} d s=2 u^{a / 2} K_{a}(2 \sqrt{u}),
$$

where $K_{a}$ denotes the $K$-Bessel function of order $a$. Also,

$$
\frac{1}{2 \pi i} \int_{\left(-\frac{1}{2}-2 M\right)} \Gamma(s+m) u^{-s} d s=u^{m} e^{-u} .
$$

Thus,

$$
I_{m, a}(x)=I_{m, a}^{+}(x)+I_{m, a}^{-}(x),
$$

where

$$
I_{m, a}^{ \pm}(x)=(2 \pi x)^{1+a / 2} e^{ \pm \pi i a / 4} \int_{0}^{\infty} u^{m+a / 2} K_{a}\left(2 e^{ \pm \pi i / 4} \sqrt{2 \pi x u}\right) e^{-u} d u .
$$


Now, for $|\arg z|<\frac{3}{2} \pi$

$$
K_{a}(z)=\sqrt{\frac{\pi}{2 z}} e^{-z}\left(1+\frac{4 a^{2}-1}{8 z}+O_{a}\left(\frac{1}{|z|^{2}}\right)\right),
$$

as $z \rightarrow \infty$, and

$$
K_{-a}(z)=K_{a}(z) \sim \begin{cases}2^{a-1} \Gamma(a) z^{-a} & \text { if } \operatorname{Re}(a) \geq 0, a \neq 0, \\ -\log (x / 2)-\gamma & \text { if } a=0\end{cases}
$$

as $z \rightarrow 0$. Therefore, by Lemma 1 ,

$$
\begin{aligned}
I_{m, a}^{ \pm}(x)=(2 \pi x)^{1+\frac{a}{2}} \frac{\pi^{\frac{1}{4}} e^{ \pm \pi i\left(a-\frac{1}{2}\right) / 4}}{2^{\frac{5}{4}} x^{\frac{1}{4}}} \int_{0}^{\infty} u^{m+\frac{a}{2}-\frac{1}{4}} e^{-u-2(1 \pm i) \sqrt{\pi x u}} \\
\times\left(1+\frac{4 a^{2}-1}{2^{\frac{9}{2}} \pi^{\frac{1}{2}} e^{ \pm \frac{\pi i}{4}} \sqrt{x u}}+O_{a}\left(\frac{1}{u}\right)\right) d u \\
\begin{aligned}
\sim 2^{\frac{1}{4}+\frac{a}{2}} \pi^{\frac{7}{4}+\frac{a}{2}} e^{ \pm \pi i\left(a-\frac{1}{2}\right) / 4} x^{\frac{3}{4}+\frac{a}{2}} e^{ \pm i \pi x} e^{-2(1 \pm i) \sqrt{\pi x n}} e^{-m} m^{m+\frac{1}{4}+\frac{a}{2}} \\
\times\left(1+\frac{\xi^{ \pm}}{\sqrt{m}}+O\left(\frac{1}{m}\right)\right),
\end{aligned}
\end{aligned}
$$

where

$$
\xi^{ \pm}=-\frac{(1 \pm i) \sqrt{\pi x}(1+a)}{2}+\frac{(1 \mp i)(\pi x)^{\frac{3}{2}}}{6}+\frac{\left(4 a^{2}-1\right)(1 \mp i)}{32 \pi^{\frac{1}{2}} \sqrt{x}},
$$

and (8) follows.

\section{An extension of Voronoi's formula}

Formula (10) can be proved with the same techniques used to prove Theorems 1 and 2. In this section we give an application of this formula and we discuss a similar formula for convolutions of the exponential function. We conclude the section showing how these results can be used to prove Voronoi's formula.

Applying formula (10) to $F(s)=\Gamma(s / 2) / 2 \Gamma(s)$ we get, for $\frac{1}{4} \pi<\arg (z)<\frac{3}{4} \pi$,

$$
\sum_{n=1}^{\infty} d(n) e^{(2 \pi n z)^{2}}=\frac{1}{z} \sum_{n=1}^{\infty} d(n) T(4 \pi n z)+R(z)+k(z),
$$

where, in the same range of $\arg (z)$,

$$
T(z):=\frac{1}{\sqrt{\pi} i} \int_{(2)} \frac{\Gamma(s)}{\Gamma(1-s / 2)}(-i z)^{-s} d s=\sum_{n=0}^{\infty} \frac{(i z)^{n}}{n ! \Gamma(1+n / 2)}
$$


and

$$
\begin{aligned}
R(z) & :=\frac{1}{4}+\frac{2 \log (-4 \pi i z)-3 \gamma}{8 \sqrt{\pi} i z}, \\
k(z) & :=\frac{1}{4 \pi^{2}} \int_{\left(-\frac{1}{2}\right)} \Gamma(s / 2) \Gamma(1-s) \zeta(s) \zeta(1-s) z^{-s} d s .
\end{aligned}
$$

Notice that we have $T(z) \ll|z|^{-B}$ for all fixed $B>0$; moreover, $k(z)$ is holomorphic in $|\arg (z)|<\frac{3}{4} \pi$ and, if $|\arg (\tau)|<\frac{1}{4} \pi$,

$$
c_{\tau}(m):=\frac{k^{(m)}(\tau)}{m !} \ll|\tau|^{-m} m^{-B}
$$

for all $B>0$. In particular, if we set $z=i \delta$ with $0<\delta \leq 1$, taking the real part of (20) we get

$$
\sum_{n=1}^{\infty} d(n) e^{-(2 \pi n \delta)^{2}}=\frac{1}{4}+\frac{-2 \log (4 \pi \delta)-3 \gamma}{4 \sqrt{\pi} \delta}+\operatorname{Re} \sum_{m=0}^{\infty} c_{m}\left(\frac{\sqrt{3}}{2}+i\left(\frac{1}{2}-\delta\right)\right)^{m}
$$

with

$$
c_{m}:=c_{(\sqrt{3}+i) / 2}(m) \ll m^{-B}
$$

for all $B>0$.

We now state a similar formula for convolutions of the exponential function and a function that is compactly supported on $\mathbb{R}_{>0}$.

Let $g(x)$ be a compactly supported function on $\mathbb{R}_{>0}$ and let

$$
W_{+}(z):=\int_{0}^{\infty} f(1 / x) \mathrm{e}(z x) \frac{d x}{x} \quad \text { and } \quad W_{-}(z):=\int_{0}^{\infty} f(x) \mathrm{e}(z x) d x .
$$

If we denote the Mellin transform of $f(x)$ with $F(s)$, then it follows that $F(s)$ is entire and that $W_{+}(x)$ and $W_{-}(x)$ can be written as in (9). In particular, since

$$
F(0)=\int_{0}^{\infty} f(x) \frac{d x}{x}, \quad F(1)=\int_{0}^{\infty} f(x) d x, \quad F^{\prime}(1)=\int_{0}^{\infty} f(x) \log x d x,
$$

formula (10) can be written as

$$
\begin{aligned}
\sum_{n=1}^{\infty} d(n) W_{+}(n z)-\frac{1}{z} \sum_{n=1}^{\infty} d(n) W_{-}(-n / z) & \\
=\int_{0}^{\infty} f(x)\left(\frac{1}{4 x}\right. & \left.-\frac{1}{4 z}-\frac{\gamma-\log (2 \pi z / x)}{2 \pi i z}\right) d x+k(z) \\
& +\int_{0}^{\infty} f(x) \int_{\left(-\frac{1}{2}\right)} \frac{\zeta(s) \zeta(1-s)}{\sin \pi s}\left(\frac{z}{x}\right)^{-s} d s \frac{d x}{2 \pi x}
\end{aligned}
$$

for $\operatorname{Im}(z)>0$. 
Proof of Voronoi's formula. Let $f: \mathbb{R}_{\geq 0} \rightarrow \mathbb{R}$ be a smooth function that decays faster than any power of $x$ and let

$$
\tilde{f}(x):=2 \int_{0}^{\infty} f(y) \cos (2 \pi x y) d y
$$

be the cosine transform of $f(x)$. Then, $\tilde{f}(x)$ is smooth and, by partial integration, $\tilde{f}^{(m)}(x) \ll 1 / x^{2+m}$ for all $m \geq 0$. For $0<\operatorname{Re}(s)<2$, we can define the Mellin transform of $\tilde{f}$,

$$
F(s):=\int_{0}^{\infty} \tilde{f}(x) x^{s-1} d x
$$

By partial integration we see that $F(s)$ extends to a meromorphic function on $\operatorname{Re}(s)<2$ with simple poles at most at the nonpositive integers. Also, $F(s)$ decays rapidly on vertical strips. Moreover, by Parseval's formula, for $0<\operatorname{Re}(s)<1$ we have

$$
\begin{aligned}
F(s) & =\frac{2}{s} \int_{0}^{\infty} f(y)(2 \pi y)^{-s} \Gamma(s+1) \cos \left(\frac{\pi s}{2}\right) d y \\
& =\frac{2}{s} \int_{0}^{\infty} f(y) d y-2 \int_{0}^{\infty} f(y)(\log (2 \pi y)+\gamma) d y+O(|s|) \\
& =\frac{F_{-1}}{s}+F_{0}+O(|s|),
\end{aligned}
$$

say. For $\operatorname{Im}(z) \geq 0$ we can define

$$
\begin{aligned}
W_{+}(z) & :=\frac{1}{2 \pi i} \int_{\left(\frac{3}{2}\right)} F(s) \Gamma(s)(-2 \pi i z)^{-s} d s=\int_{0}^{\infty} \tilde{f}\left(\frac{1}{x}\right) \mathrm{e}(z x) \frac{d x}{x}, \\
W_{-}(z) & :=\frac{1}{2 \pi i} \int_{\left(\frac{3}{2}\right)} F(1-s) \Gamma(s)(-2 \pi i z)^{-s} d s \\
& =\int_{0}^{\infty}\left(\tilde{f}(x)-\operatorname{Res}_{s=0} F(s)\right) \mathrm{e}(z x) d x
\end{aligned}
$$

with the second representation of $W_{-}(z)$ defined only on $\operatorname{Im}(z)>0$. Since $F(s)$ is rapidly decaying at infinity, (10) holds for $\operatorname{Im}(z) \geq 0$ and so we can apply that formula for $z=1$ and take the real part. By the definition of $\tilde{f}$, we have

$$
\begin{aligned}
\operatorname{Re}\left(W_{+}(n)\right) & =2 \int_{0}^{\infty} f(y) \int_{0}^{\infty} \cos \left(\frac{2 \pi y}{x}\right) \cos (n x) \frac{d x}{x} d y \\
& =\int_{0}^{\infty} f(y)\left(2 K_{0}(4 \pi \sqrt{n y})-\pi Y_{0}(4 \pi \sqrt{n y})\right) d y
\end{aligned}
$$


and

$$
\begin{aligned}
\operatorname{Re}\left(W_{-}(-n)\right) & =\lim _{\substack{z \rightarrow 1, \operatorname{Im}(z)>0}} \operatorname{Re}\left(W_{-}(-n z)\right) \\
& =\lim _{\substack{z \rightarrow 1, \operatorname{Im}(z)>0}} \operatorname{Re} \int_{0}^{\infty} \tilde{f}(x) \mathrm{e}(-n z x) d x-\lim _{\substack{z \rightarrow 1, \operatorname{Im}(z)>0}} \operatorname{Re} \frac{\operatorname{Res}_{s=0} F(s)}{-2 \pi i n z} \\
& =\frac{1}{2} f(n),
\end{aligned}
$$

since $\operatorname{Res}_{s=0} F(s)$ is real. Moreover, $(2 \pi)^{-1} \int_{(-1 / 2)} F(s) \frac{\zeta(s) \zeta(1-s)}{\sin \pi s} z^{-s} d s$ is purely imaginary on the real line, so we just need to compute

$$
\begin{aligned}
\operatorname{Re}\left(\operatorname{Res}_{s=0,1}\right. & \left.F(s) \Gamma(s) \zeta(s)^{2}(-2 \pi i)^{-s}\right) \\
& =\operatorname{Re}\left(\frac{F(1)(\gamma-\log (-2 \pi i))+F^{\prime}(1)}{-2 \pi i}\right. \\
& \left.+\frac{-F_{-1}(\log (-2 \pi i)+\gamma-2 \log 2 \pi)+F_{0}}{4}\right) \\
& =-\frac{f(0)}{8}-\frac{1}{2} \int_{0}^{\infty} f(y)(\log y+2 \gamma) d y,
\end{aligned}
$$

since $F(1)=f(0) / 2$ and $F^{\prime}(1)$ is real. This completes the proof of the theorem.

\section{An exact formula for the second moment of $\zeta(s)$}

In this section we prove the exact formula for the second moment of the Riemann zeta function.

Proof of Theorem 3. Firstly, observe that

$$
L_{2}(\delta)=-i e^{-i \delta / 2} \int_{\frac{1}{2}}^{\frac{1}{2}+i \infty} \zeta(s) \zeta(1-s) e^{i \delta s} d s .
$$

The functional equation for $\zeta(s)$,

$$
\zeta(1-s)=\chi(1-s) \zeta(s)
$$

where

$$
\chi(1-s)=(2 \pi)^{-s} \Gamma(s)\left(e^{\pi i s / 2}+e^{-\pi i s / 2}\right),
$$

allows us to split $L_{2}(\delta)$ as

$$
L_{2}(\delta)=-i e^{-i \delta / 2} \int_{\frac{1}{2}}^{\frac{1}{2}+i \infty} \chi(1-s) \zeta(s)^{2} e^{i \delta s} d s=-i e^{-i \delta / 2}\left(L^{+}(\delta)+L^{-}(\delta)\right),
$$


where

$$
L^{ \pm}(\delta)=\int_{\frac{1}{2}}^{\frac{1}{2}+i \infty}(2 \pi)^{-s} \Gamma(s) e^{ \pm \pi i s / 2} \zeta(s)^{2} e^{i \delta s} d s .
$$

By Stirling's formula, $L^{+}(\delta)$ is analytic for $\operatorname{Re}(\delta)>-\pi$. Moreover, by contour integration,

$$
L^{-}(\delta)=\int_{(2)}(2 \pi)^{-s} \Gamma(s) e^{-\pi i s / 2} \zeta(s)^{2} e^{i \delta s} d s-G(\delta)=J(\delta)-G(\delta),
$$

say, where

$$
\begin{aligned}
G(\delta):=\int_{\frac{1}{2}-i \infty}^{\frac{1}{2}}(2 \pi)^{-s} \Gamma(s) e^{-\pi i s / 2} \zeta(s)^{2} e^{i \delta s} d s & \\
& +2 \pi i \operatorname{Res}_{s=1}\left((2 \pi)^{-s} \Gamma(s) e^{-\pi i s / 2} \zeta(s)^{2} e^{i \delta s}\right)
\end{aligned}
$$

is analytic for $\operatorname{Re}(\delta)<\pi$. Now, expanding $\zeta(s)^{2}$ into its Dirichlet series, for $\operatorname{Re}(\delta)>0$ we have

$$
\begin{aligned}
J(\delta) & =\sum_{n=1}^{\infty} d(n) \int_{2-i \infty}^{2+i \infty} \Gamma(s)\left(2 \pi i n e^{-i \delta}\right)^{-s} d s \\
& =2 \pi i \mathscr{Y}_{0}\left(-e^{-i \delta}\right)=2 \pi i \mathscr{Y}_{0}\left(1-e^{-i \delta}\right) .
\end{aligned}
$$

By Theorem 1, we can write this as

$$
J(\delta)=\frac{\log 2 \pi \delta-\gamma}{1-e^{-i \delta}}-\pi g_{0}\left(1-e^{-i \delta}\right)+\frac{2 \pi i}{1-e^{-i \delta}} \mathscr{Y}_{0}\left(\frac{-1}{1-e^{-i \delta}}\right)+i e^{i \delta} \omega(\delta),
$$

where

$$
\omega(\delta)=-\frac{\log \left(\left(1-e^{-i \delta}\right) / \delta\right)-\frac{1}{2} \pi i}{2 \sin (\delta / 2)}
$$

is holomorphic in $|\operatorname{Re}(\delta)|<\pi$. Summing up, we have

$$
\begin{array}{r}
L_{2}(\delta)=\frac{\gamma-\log 2 \pi \delta}{2 \sin (\delta / 2)}+\frac{\pi i}{\sin (\delta / 2)} \varphi_{0}\left(\frac{-1}{1-e^{-i \delta}}\right)+i \pi e^{-i \delta / 2} g_{0}\left(1-e^{-i \delta}\right) \\
+\omega(\delta)-i e^{-i \delta / 2}\left(L^{+}(\delta)-G(\delta)\right) .
\end{array}
$$

The theorem then follows after writing

$$
h(\delta):=i \pi e^{-i \delta / 2} g_{0}\left(1-e^{-i \delta}\right)
$$

and applying Theorems 1 and 2. 


\section{Cotangent sums}

We start by recalling the basic properties of $D(s, a, h / k)$.

Lemma 2. For $(h, k)=1, k>0$ and $a \in \mathbb{C}$,

$$
D\left(s, a, \frac{h}{k}\right)-k^{1+a-2 s} \zeta(s-a) \zeta(s)
$$

is an entire function of $s$. Moreover, $D(s, a, h / k)$ satisfies a functional equation,

$$
\begin{aligned}
& D\left(s, a, \frac{h}{k}\right) \\
& =-\frac{2}{k}\left(\frac{k}{2 \pi}\right)^{2-2 s+a} \Gamma(1-s+a) \Gamma(1-s) \\
& \quad \times\left(\cos \left(\frac{\pi}{2}(2 s-a)\right) D\left(1-s,-a,-\frac{\bar{h}}{k}\right)-\cos \frac{\pi a}{2} D\left(1-s,-a, \frac{\bar{h}}{k}\right)\right),
\end{aligned}
$$

and

$$
D\left(0, a, \frac{h}{k}\right)=\frac{i}{2} c_{a}\left(\frac{h}{k}\right)-\frac{1}{2} \zeta(-a) .
$$

Proof. The analytic continuation and the functional equation for $D(s, a, h / k)$ can be proved easily using the analogous properties for the Hurwitz zeta function and the observation that

$$
D\left(s, a, \frac{h}{k}\right)=\frac{1}{k^{2 s-a}} \sum_{m, n=1}^{k} \mathrm{e}\left(\frac{m n h}{k}\right) \zeta\left(s-a, \frac{m}{k}\right) \zeta\left(s, \frac{n}{k}\right) .
$$

Moreover, applying this equality at 0 , we see that

$$
\begin{aligned}
D\left(0, a, \frac{h}{k}\right) & =-k^{a} \sum_{m, n=1}^{k-1} \mathrm{e}\left(\frac{m n h}{k}\right) \zeta\left(-a, \frac{m}{k}\right) B_{1}\left(\frac{n}{k}\right)-\frac{\zeta(-a)}{2} \\
& =\frac{i}{2} c_{a}\left(\frac{h}{k}\right)-\frac{\zeta(-a)}{2}
\end{aligned}
$$

where we used

$$
\sum_{n=1}^{k-1} B_{1}\left(\frac{n}{k}\right)\left(\mathrm{e}\left(\frac{m h}{k}\right)\right)^{n}=-\frac{1}{2} \frac{1+\mathrm{e}\left(\frac{m h}{k}\right)}{1-\mathrm{e}\left(\frac{m h}{k}\right)}=-\frac{i}{2} \cot \left(\frac{\pi m h}{k}\right)
$$

which can be easily obtained from the equality

$$
B_{1}(x)=\left.\frac{\mathrm{d}}{\mathrm{d} t}\left(\frac{t e^{x t}}{e^{t}-1}\right)\right|_{t=0} .
$$

Proof of Theorem 4. First observe that we can assume $0 \neq|a|<1$, since the result extends to all $a$ by analytic continuation. Now, taking $z=\frac{h}{k}(1+i \delta)$, with $\delta>0$, 
we have

$$
\begin{aligned}
\mathscr{S}_{a}(z) & =\sum_{n \geq 1} \sigma_{a}(n) \mathrm{e}\left(n \frac{h}{k}\right) e^{-2 \pi n(h / k) \delta} \\
& =\frac{1}{2 \pi i} \int_{(2)} \Gamma(s) D\left(s, a, \frac{h}{k}\right)\left(2 \pi \frac{h}{k} \delta\right)^{-s} d s .
\end{aligned}
$$

Therefore, moving the integral to $\sigma=-\frac{1}{2}$,

$$
\mathscr{S}_{a}(z)=\frac{k^{a}}{2 \pi h \delta} \zeta(1-a)+\frac{1}{(2 \pi h \delta)^{1+a}} \zeta(1+a) \Gamma(1+a)+D\left(0, a, \frac{h}{k}\right)+O\left(\delta^{1 / 2}\right) .
$$

Similarly,

$$
\begin{aligned}
\frac{1}{z^{1+a}} \mathscr{Y}_{a}\left(\frac{-1}{z}\right)= & \frac{1}{z^{1+a}} \sum_{n \geq 1} \sigma_{a}(n) \mathrm{e}\left(-n \frac{k}{h}\right) e^{-2 \pi n(k / h) \delta /(1+i \delta)} \\
= & \frac{k^{a}}{2 \pi \delta h} \zeta(1-a)+\frac{1}{(2 \pi \delta h)^{1+a} \zeta(1+a) \Gamma(1+a)} \\
& \quad-i a \frac{k^{a}}{2 \pi h} \zeta(1-a)+\left(\frac{k}{h(1+i \delta)}\right)^{1+a} D\left(0, a,-\frac{k}{h}\right)+O\left(\delta^{1 / 2}\right) .
\end{aligned}
$$

In particular, as $\delta$ goes to 0 , we have

$\mathscr{S}_{a}(z)-\frac{1}{z^{1+a}} \mathscr{Y}_{a}\left(\frac{-1}{z}\right) \longrightarrow D\left(0, a, \frac{h}{k}\right)-\left(\frac{k}{h}\right)^{1+a} D\left(0, a,-\frac{k}{h}\right)+i a \frac{k^{a}}{2 \pi h} \zeta(1-a)$.

Applying Theorem 1, it follows that

$$
\begin{aligned}
D\left(0, a, \frac{h}{k}\right)-\left(\frac{k}{h}\right)^{1+a} D\left(0, a,-\frac{k}{h}\right)+i a \frac{k^{a}}{2 \pi h} \zeta & (1-a) \\
& =\frac{\zeta(-a)}{2}\left(\left(\frac{k}{h}\right)^{1+a}-1+\psi_{a}\left(\frac{h}{k}\right)\right),
\end{aligned}
$$

which is equivalent to (17).

We conclude the paper by giving a new proof of Vasyunin's formula (with a shift).

Theorem 5. Let $(h, k)=1$, with $h, k \geq 1$. Let $|\operatorname{Re}(a)|<1$. Then

$$
\begin{array}{r}
\frac{1+a}{2 \pi} \int_{-\infty}^{\infty} \zeta\left(\frac{1}{2}+\frac{a}{2}+i t\right) \zeta\left(\frac{1}{2}+\frac{a}{2}-i t\right)\left(\frac{h}{k}\right)^{-i t} \frac{d t}{\left(\frac{1}{2}+\frac{a}{2}+i t\right)\left(\frac{1}{2}+\frac{a}{2}-i t\right)} \\
=-\frac{\zeta(1+a)}{2}\left(\left(\frac{k}{h}\right)^{\frac{1}{2}+\frac{a}{2}}+\left(\frac{h}{k}\right)^{\frac{1}{2}+\frac{a}{2}}\right)-\frac{\zeta(a)}{a}\left(\left(\frac{k}{h}\right)^{\frac{1}{2}-\frac{a}{2}}+\left(\frac{h}{k}\right)^{\frac{1}{2}-\frac{a}{2}}\right) \\
-\left(\frac{1}{h k}\right)^{\frac{1}{2}+\frac{a}{2}}(2 \pi)^{a} \Gamma(-a) \sin \frac{\pi a}{2}\left(c_{a}\left(\frac{\bar{h}}{k}\right)+c_{a}\left(\frac{\bar{k}}{h}\right)\right) .
\end{array}
$$


Proof. We need to evaluate

$$
\begin{aligned}
& \frac{1+a}{2 \pi(h k)^{\frac{1}{2}+\frac{a}{2}}} \int_{-\infty}^{\infty} \zeta\left(\frac{1}{2}+\frac{a}{2}+i t\right) \zeta\left(\frac{1}{2}+\frac{a}{2}-i t\right)\left(\frac{h}{k}\right)^{i t} \frac{d t}{\left(\frac{1}{2}+\frac{a}{2}+i t\right)\left(\frac{1}{2}+\frac{a}{2}-i t\right)} \\
& =\frac{1+a}{2 \pi i} \int_{\left(\frac{1}{2}-\frac{\operatorname{Re}(a)}{2}\right)} \frac{\zeta(s+a) \zeta(1-s)}{h^{s+a} k^{1-s}} \frac{d s}{(s+a)(1-s)} .
\end{aligned}
$$

We rewrite this as

$$
\begin{aligned}
& \frac{1+a}{2 \pi i} \int_{\left(\frac{1}{2}-\frac{\operatorname{Re}(a)}{2}\right)} \frac{\zeta(s+a) \zeta(1-s)}{h^{s+a} k^{1-s}} \frac{d s}{(s+a)(1-s)} \\
& =\frac{1}{2 \pi i} \int_{\left(\frac{1}{2}-\frac{\operatorname{Re}(a)}{2}\right)} \frac{\zeta(s+a) \zeta(1-s)}{h^{s+a} k^{1-s}} \frac{d s}{1-s}+\frac{1}{2 \pi i} \int_{\left(\frac{1}{2}-\frac{\operatorname{Re}(a)}{2}\right)} \frac{\zeta(s+a) \zeta(1-s)}{h^{s+a} k^{1-s}} \frac{d s}{s+a} \\
& =I_{a}\left(\frac{h}{k}\right)+I_{a}\left(\frac{k}{h}\right),
\end{aligned}
$$

where

$$
I_{a}\left(\frac{h}{k}\right):=\frac{1}{2 \pi i} \int_{\left(\frac{1}{2}-\frac{\operatorname{Re}(a)}{2}\right)} \frac{\zeta(s+a) \zeta(1-s)}{h^{s+a} k^{1-s}} \frac{d s}{1-s} .
$$

The integral is not absolutely convergent, so some care is needed. One could introduce a convergence factor $e^{\delta s^{2}}$ and let $\delta \rightarrow 0^{+}$at the end of the argument, or one could work with the understanding that the integrals are to be interpreted as $\lim _{T \rightarrow \infty} \int_{c-i T}^{c+i T}$. We opt for the latter. Recall that $\zeta(s)=\chi(s) \zeta(1-s)$, where

$$
\chi(1-s)=\left((-2 \pi i)^{-s}+(2 \pi i)^{-s}\right) \Gamma(s) .
$$

This leads to

$$
\begin{aligned}
\frac{1}{2 \pi i} \int_{(2)} \frac{\chi(1-s)}{1-s} & u^{-s} d s=\frac{-1}{2 \pi i} \int_{(2)}\left((-2 \pi i)^{-s}+(2 \pi i)^{-s}\right) \frac{\Gamma(s)}{s-1} u^{-s} d s \\
& =\frac{-1}{2 \pi i u} \int_{(1)}\left((-2 \pi i)^{-s-1}+(2 \pi i)^{-s-1}\right) \Gamma(s) u^{-s} d s=\frac{\sin 2 \pi u}{\pi u} .
\end{aligned}
$$

Using Cauchy's theorem, the functional equation for $\zeta(s)$, and the Dirichlet series for $\zeta(s+a) \zeta(s)$, we have

$$
\begin{aligned}
I_{a}\left(\frac{h}{k}\right)=-\operatorname{Res}_{s=1} \frac{\chi(1-s) \zeta(s+a) \zeta(s)}{h^{s+a} k^{1-s}(1-s)}-\operatorname{Res}_{s=1-a} \frac{\chi(1-s) \zeta(s+a) \zeta(s)}{h^{s+a} k^{1-s}(1-s)} \\
+\frac{1}{\pi h^{1+a}} \sum_{n=1}^{\infty} \frac{\sigma_{-a}(n) \sin \left(2 \pi n \frac{h}{k}\right)}{n} \\
=-\frac{\zeta(1+a)}{2 h^{1+a}}-\frac{\zeta(a)}{a h k^{a}}+\frac{1}{\pi h^{1+a}} \sum_{n=1}^{\infty} \frac{\sigma_{-a}(n) \sin \left(2 \pi n \frac{h}{k}\right)}{n}
\end{aligned}
$$


By the functional equation for $D$ we see that

$$
\begin{aligned}
\frac{D\left(s,-a, \frac{h}{k}\right)-D\left(s,-a,-\frac{h}{k}\right)}{2 i} & =\frac{2}{k}\left(\frac{k}{2 \pi}\right)^{2-2 s-a} \Gamma(1-s-a) \Gamma(1-s) \\
\times\left(\cos \left(\frac{\pi}{2}(2 s+a)\right)\right. & \left.+\cos \frac{\pi a}{2}\right)\left(D\left(1-s, a, \frac{\bar{h}}{k}\right)-D\left(1-s, a,-\frac{\bar{h}}{k}\right)\right),
\end{aligned}
$$

so that, defining

$$
S\left(s,-a, \frac{h}{k}\right):=\sum_{n=1}^{\infty} \frac{\sigma_{-a}(n) \sin \left(2 \pi n \frac{h}{k}\right)}{n^{s}},
$$

we have

$$
\begin{aligned}
& S\left(s,-a, \frac{h}{k}\right)=\frac{2}{k}\left(\frac{k}{2 \pi}\right)^{2-2 s-a} \Gamma(1-s-a) \Gamma(1-s) \\
& \times\left(\cos \left(\frac{\pi}{2}(2 s+a)\right)+\cos \frac{\pi a}{2}\right) S\left(1-s, a, \frac{\bar{h}}{k}\right) .
\end{aligned}
$$

In particular, $S\left(s,-a, \frac{h}{k}\right)$ is regular at $s=1$. Noting that

$$
\lim _{s \rightarrow 1} \Gamma(1-s-a) \Gamma(1-s)\left(\cos \left(\frac{\pi}{2}(2 s+a)\right)+\cos \frac{\pi a}{2}\right)=-\pi \Gamma(-a) \sin \frac{\pi a}{2}
$$

and

$$
S(0, a, \bar{h} / k)=\frac{1}{2} c_{a}(\bar{h} / k),
$$

we obtain, by letting $s \rightarrow 1$ in (27), the identity

$$
S\left(1,-a, \frac{h}{k}\right)=2^{a}\left(\frac{\pi}{k}\right)^{1+a} \Gamma(-a) \sin \frac{\pi a}{2} c_{a}\left(\frac{\bar{h}}{k}\right),
$$

whence

$$
\sum_{n=1}^{\infty} \frac{\sigma_{-a}(n) \sin \left(2 \pi n \frac{h}{k}\right)}{\pi n h^{1+a}}=-\left(\frac{1}{h k}\right)^{1+a}(2 \pi)^{a} \Gamma(-a) \sin \frac{\pi a}{2} c_{a}\left(\frac{\bar{h}}{k}\right) .
$$

Thus,

$$
I_{a}\left(\frac{h}{k}\right)=-\frac{\zeta(1+a)}{2 h^{1+a}}-\frac{\zeta(a)}{a h k^{a}}-\left(\frac{1}{h k}\right)^{1+a}(2 \pi)^{a} \Gamma(-a) \sin \frac{\pi a}{2} c_{a}\left(\frac{\bar{h}}{k}\right)
$$

and the theorem follows.

\section{References}

[Báez-Duarte et al. 2005] L. Báez-Duarte, M. Balazard, B. Landreau, and E. Saias, "Étude de l'autocorrélation multiplicative de la fonction 'partie fractionnaire", Ramanujan J. 9:1-2 (2005), 
[Bagchi 2006] B. Bagchi, "On Nyman, Beurling and Baez-Duarte's Hilbert space reformulation of the Riemann hypothesis", Proc. Indian Acad. Sci. Math. Sci. 116:2 (2006), 137-146. MR 2007b: 11126 Zbl 1125.11049

[Balasubramanian et al. 1985] R. Balasubramanian, J. B. Conrey, and D. R. Heath-Brown, "Asymptotic mean square of the product of the Riemann zeta-function and a Dirichlet polynomial", J. Reine Angew. Math. 357 (1985), 161-181. MR 87f:11061 Zbl 0549.10030

[Bettin and Conrey 2011] S. Bettin and J. Conrey, "A reciprocity formula for a cotangent sum", preprint, 2011.

[Bruggeman 2007] R. Bruggeman, "Quantum Maass forms", pp. 1-15 in The conference on Lfunctions (Fukuoka, 2006), edited by L. Weng and M. Kaneko, World Scientific Publishing, Hackensack, NJ, 2007. MR 2008g:11076 Zbl 1130.11019

[Conrey 1989] J. B. Conrey, "More than two fifths of the zeros of the Riemann zeta function are on the critical line", J. Reine Angew. Math. 399 (1989), 1-26. MR 90g:11120 Zbl 0668.10044

[Conrey and Ghosh 1998] J. B. Conrey and A. Ghosh, "A conjecture for the sixth power moment of the Riemann zeta-function”, Internat. Math. Res. Notices 1998:15 (1998), 775-780. MR 99h:11096 Zbl 0920.11060

[Conrey and Gonek 2001] J. B. Conrey and S. M. Gonek, "High moments of the Riemann zetafunction”, Duke Math. J. 107:3 (2001), 577-604. MR 2002b:11112 Zbl 1006.11048

[Hardy and Littlewood 1916] G. H. Hardy and J. E. Littlewood, "Contributions to the theory of the Riemann zeta-function and the theory of the distribution of primes", Acta Math. 41:1 (1916), 119-196. MR 1555148 JFM 46.0498.01

[Ingham 1927] A. E. Ingham, "Mean-value theorems in the theory of the Riemann zeta-function", Proc. London Math. Soc. (2) 27:1 (1927), 273. MR 1575391 JFM 53.0313.01

[Iwaniec 1980] H. Iwaniec, "On mean values for Dirichlet's polynomials and the Riemann zeta function”, J. London Math. Soc. (2) 22:1 (1980), 39-45. MR 81m:10076 Zbl 0439.10026

[Keating and Snaith 2000] J. P. Keating and N. C. Snaith, "Random matrix theory and $\zeta(1 / 2+i t)$ ", Comm. Math. Phys. 214:1 (2000), 57-89. MR 2002c:11107 Zbl 1051.11048

[Landreau and Richard 2002] B. Landreau and F. Richard, "Le critère de Beurling et Nyman pour l'hypothèse de Riemann: aspects numériques", Experiment. Math. 11:3 (2002), 349-360. MR 2004c:11159 Zbl 1117.11305

[Lewis and Zagier 2001] J. Lewis and D. Zagier, "Period functions for Maass wave forms, I", Ann. of Math. (2) 153:1 (2001), 191-258. MR 2003d:11068 Zbl 1061.11021

[Motohashi 1997] Y. Motohashi, Spectral theory of the Riemann zeta-function, Cambridge Tracts in Mathematics 127, Cambridge University Press, 1997. MR 99f:11109 Zbl 0878.11001

[Zagier 2010] D. Zagier, “Quantum modular forms”, pp. 659-675 in Quanta of maths (Paris, 2007), edited by E. Blanchard et al., Clay Math. Proc. 11, American Mathematical Society, Providence, RI, 2010. MR 2012a:11066 Zbl 05902011

Communicated by Barry Mazur

Received 2011-12-01 Revised 2012-01-15 Accepted 2012-02-20

sandro.bettin@gmail.com

School of Mathematics, University of Bristol, Howard House, Queens Avenue, Bristol BS82NF, United Kingdom http://www. maths.bris.ac.uk/ maxsb/

conrey@aimath.org

American Institute of Mathematics, 360 Portage Avenue, Palo Alto, CA 94306, United States 


\section{Algebra \& Number Theory}

msp.org/ant

\section{EDITORS}

MANAGING EDITOR

Bjorn Poonen

Massachusetts Institute of Technology

Cambridge, USA

\author{
EDITORIAL BOARD CHAIR \\ David Eisenbud \\ University of California \\ Berkeley, USA
}

\section{BOARD OF EDITORS}

Georgia Benkart

Dave Benson

Richard E. Borcherds

John H. Coates

J-L. Colliot-Thélène

Brian D. Conrad

Hélène Esnault

Hubert Flenner

Edward Frenkel

Andrew Granville

Joseph Gubeladze

Ehud Hrushovski

Craig Huneke

Mikhail Kapranov

Yujiro Kawamata

János Kollár

Yuri Manin

Barry Mazur

Philippe Michel
University of Wisconsin, Madison, USA

University of Aberdeen, Scotland

University of California, Berkeley, USA

University of Cambridge, UK

CNRS, Université Paris-Sud, France

University of Michigan, USA

Freie Universität Berlin, Germany

Ruhr-Universität, Germany

University of California, Berkeley, USA

Université de Montréal, Canada

San Francisco State University, USA

Hebrew University, Israel

University of Virginia, USA

Yale University, USA

University of Tokyo, Japan

Princeton University, USA

Northwestern University, USA

Harvard University, USA

École Polytechnique Fédérale de Lausanne
Susan Montgomery

Shigefumi Mori

Raman Parimala

Jonathan Pila

Victor Reiner

Karl Rubin

Peter Sarnak

Joseph H. Silverman

Michael Singer

Vasudevan Srinivas

J. Toby Stafford

Bernd Sturmfels

Richard Taylor

Ravi Vakil

Michel van den Bergh

Marie-France Vignéras

Kei-Ichi Watanabe

Andrei Zelevinsky

Efim Zelmanov
University of Southern California, USA

RIMS, Kyoto University, Japan

Emory University, USA

University of Oxford, UK

University of Minnesota, USA

University of California, Irvine, USA

Princeton University, USA

Brown University, USA

North Carolina State University, USA

Tata Inst. of Fund. Research, India

University of Michigan, USA

University of California, Berkeley, USA

Harvard University, USA

Stanford University, USA

Hasselt University, Belgium

Université Paris VII, France

Nihon University, Japan

Northeastern University, USA

University of California, San Diego, USA

\section{PRODUCTION}

production@msp.org

Silvio Levy, Scientific Editor

See inside back cover or msp.org/ant for submission instructions.

The subscription price for 2013 is US \$200/year for the electronic version, and \$350/year ( $\$ 40$, if shipping outside the US) for print and electronic. Subscriptions, requests for back issues and changes of subscribers address should be sent to MSP.

Algebra \& Number Theory (ISSN 1944-7833 electronic, 1937-0652 printed) at Mathematical Sciences Publishers, 798 Evans Hall \#3840, c/o University of California, Berkeley, CA 94720-3840 is published continuously online. Periodical rate postage paid at Berkeley, CA 94704, and additional mailing offices.

ANT peer review and production are managed by EditFLOW ${ }^{\circledR}$ from Mathematical Sciences Publishers.

\section{PUBLISHED BY}

mathematical sciences publishers

nonprofit scientific publishing

http://msp.org/

(C) 2013 Mathematical Sciences Publishers 


\section{Algebra \& Number Theory}

\section{Volume $7 \quad$ No. $1 \quad 2013$}

Powers of ideals and the cohomology of stalks and fibers of morphisms

MARC CHARDIN

Graphs of Hecke operators

OLIVER LORSCHEID

Group actions of prime order on local normal rings

FrANZ KIRÀLY and WERNER LÜTKEBOHMERT

On the arithmetic and geometry of binary Hamiltonian forms

JOUNI PARKKONEN and FRÉDÉRIC PAULIN

$L$-functions and periods of adjoint motives

MICHAEL HARRIS

Galois module structure of local unit groups

ROMYAR SHARIFI

On the invariant theory for tame tilted algebras

CALIN CHINDRIS

Period functions and cotangent sums

SANDRO BETTIN and BRIAN CONREY 